\title{
La répartition des tâches entre médecins et praticiens du droit
}

\author{
Résumé de l'arrêt \\ En juin 2014, le Tribunal fédéral a rendu, dans le cadre \\ d'un cas d'assurance invalidité, un arrêt discuté dans \\ les médias. Nous n'aborderons pas ici les détails du cas \\ concerné, ni la délimitation entre troubles douloureux [qui, \\ selon la pratique judiciaire, ne sont en principe pas inva- \\ lidants] et troubles dépressifs. En effet, cet article a pour \\ sujet les constatations du Tribunal fédéral qui risquent de \\ déboucher sur des confusions au sein du corps médical. Au \\ considérant 3.2 de son arrêt, le Tribunal fédéral définit \\ comme suit la répartition des tâches et des compétences \\ dans le cadre de l'évaluation de l'invalidité: «Il incombe \\ tout d'abord au médecin (chargé de l'évaluation) d'éva-
}

luer l'état de santé et, si nécessaire, de décrire son évolution dans le temps, c'est-à-dire de réunir les résultats des investigations en procédant à un examen médical selon les règles de l'art et en tenant compte des plaintes subjectives, puis de poser un diagnostic en se fondant sur ces résultats. [...] En revanche, le médecin ne jouit pas de la compétence d'évaluer en dernier ressort les répercussions des troubles de la santé diagnostiqués sur la capacité de travail» (traduction FMH). Et le Tribunal fédéral de poursuivre: "En effet, il n'existe pas de corrélation entre le diagnostic médical et l'incapacité de travail, et ce tant en ce qui concerne les affections somatiques qu'en ce qui concerne les affections psychiques» (traduction FMH).

\section{Renato Marelli}

Président de la Société suisse de psychiatrie d'assurance SSPA
Correspondance: Dr Renato Marelli Société suisse de psychiatrie d'assurance SSPA Leonhardsstrasse 16 CH-4051 Bâle

\section{Commentaire sous l'angle de la médecine d'assurance}

Dans le cas précité, le Tribunal fédéral s'est exprimé en détail sur la répartition des tâches entre médecins et praticiens du droit dans le cadre de l'évaluation de l'incapacité de travail. A diverses occasions, des confrères se sont demandé quelles étaient désormais leurs compétences réelles au moment de délivrer une incapacité de travail, question qui a également été abordée par la presse. C'est pourquoi un commentaire de cet arrêt sous l'angle de la médecine des assurances s'impose.

\section{Incapacité de travail dans le cadre de l'évaluation de l'invalidité}

Il convient tout d'abord de souligner que l'arrêt du Tribunal fédéral précité a été rendu dans le cadre d'un cas d'évaluation de l'invalidité. Dans un tel cas, il s'agit de discuter d'une incapacité de travail de longue durée plutôt que d'une incapacité de travail aiguë. Dès lors, il s'agit de considérer l'incapacité de travail non seulement dans la profession ou le domaine de compétence actuels, mais également dans le cadre d'une autre activité, d'une autre profession, ou d'un autre domaine de compétence. L'évaluation de l'invalidité consiste à établir, du point de vue médical, tant les ressources en termes de performance que les déficits et les limitations fonctionnels dont découle l'atteinte à la capacité de travail. Il convient par ailleurs d'évaluer les chances de succès d'un traitement et de poser un pronostic en conséquence.

Il n'échappera pas au lecteur attentif de l'arrêt du Tribunal fédéral que celui-ci ne mentionne pas une étape pourtant décisive et incontournable de l'évaluation de l'incapacité de travail une fois le diagnostic posé: la personne qui procède à l'évaluation doit notamment établir quelles fonctions physiques et/ou psychiques sont limitées, dans quelle mesure et de quelle manière elles le sont, et en déduire quelles activités la personne assurée ne peut plus exercer ou uniquement de manière limitée. Il est regrettable que l'arrêt du Tribunal fédéral ne mentionne pas cette étape et risque ainsi de créer une confusion, en particulier chez les personnes qui ne sont pas souvent confrontées à des expertises médicales pour l'assurance invalidité.

Il aurait été utile que l'arrêt précité mentionne expressément cette étape afin de dissiper d'éventuels malentendus, et ce pour les trois raisons suivantes:

Premièrement, le praticien du droit n'est en mesure d'honorer véritablement sa tâche d'évaluation des conséquences sur la capacité de travail sous l'angle d'une éventuelle incapacité de gain que s'il admet qu'il existe une atteinte à la santé qui doit être diagnostiquée, et avant tout s'il comprend de quelle manière et pour quelle raison le médecin est parvenu à ses conclusions en ce qui concerne l'évaluation de la capacité de travail. Seule l'appréciation de l'acceptabilité permet, dans un second temps, de juger l'évaluation médicale sous l'angle juridique, et de la comprendre ou de la corriger. Il incombe aussi au praticien du droit d'apprécier les informations relatives à l'incapacité de travail sous l'angle juridique, si cellesci sont contradictoires ce qui intrinsèquement est tout à fait possible.

Deuxièmement, l'affirmation du Tribunal fédéral selon laquelle «il n'existe pas de corrélation entre le diagnostic médical et l'incapacité de travail», devien- 
drait intelligibles pour le lecteur qui ne dispose pas d'une formation en matière d'expertises si elle est complétée par celle selon laquelle le médecin doit établir les limitations fonctionnelles et les restrictions d'activités conditionnées par l'état de santé. En effet, il existe incontestablement des diagnostics tant somatiques que psychiatriques qui peuvent amener un profane à partir du principe que la capacité de travail de la personne concernée est clairement atteinte. En cas d'infarctus aigu du myocarde, la corrélation entre maladie et incapacité de travail est très étroite. Le syndrome paranoïaque hallucinatoire lié à la schizophrénie constitue un autre exemple de ce type. Néanmoins, même dans de tels exemples, l'incapacité de travail ne peut être attestée que grâce à la séquence causale de constatations dans le cadre du diagnostic, aux troubles des fonctions physiques ou psychiques qui en découlent et à la limitation des activités et de la participation au processus de travail qui en résulte à son tour. Ainsi, l'appréciation médicale des fonctions et des activités est indispensable dans les cas faisant l'objet d'une expertise dans le cadre de l'évaluation de l'invalidité et dans les cas sur lesquels le juge est amené à se prononcer. Dans de tels cas, le rapport entre la constellation des résultats d'examen et l'éventuelle incapacité de travail n'est pratiquement jamais aussi étroit que dans les exemples précités, ce qui signifie qu'il n'existe effectivement pas de corrélation (directe) entre diagnostic et incapacité de travail. Le Tribunal fédéral cite à cet égard une étude qui tient compte pars pro toto de ce facteur [1].

Troisièmement, une telle indication dans l'arrêt serait également un appel aux médecins qui ne se sont pas encore appropriés de façon routinière la description des limitations affectant les fonctions et les activités dans le cadre de l'attestation d'une incapacité de travail. Cet article vise donc à combler cette lacune.

\section{Incapacité de travail dans le cadre de l'assurance d'indemnités journalières ou d'un certificat destiné à l'employeur}

Les médias se sont par ailleurs penchés sur les éventuelles conséquences de l'arrêt du Tribunal fédéral sur les certificats médicaux dans le domaine de l'as- surance d'indemnités journalières. Néanmoins, l'arrêt en question ne concerne manifestement pas de tels certificats. Dans le domaine de l'assurance d'indemnités journalières, il incombe au médecin de juger en dernier ressort et en son âme et conscience de la capacité de travail. L'employeur ou l'assurance d'indemnités journalières peuvent bien entendu contester son évaluation si elle est dénuée de fondement ou si de nouveaux faits, inconnus au moment de l'évaluation, jettent le doute sur celle-ci. Toutefois, dans un tel cas, il incombe à l'employeur ou à l'assurance d'indemnités journalières de solliciter une nouvelle évaluation médicale. Seule cette dernière peut, le cas échéant, remettre en cause et/ou corriger la première évaluation médicale.

1 Klipstein, Michel, Läubli et al. Do MRI findings correlate with mobility tests? Eur Spine. 2007;803-11.

\section{Résumé et attentes}

En résumé, l'arrêt précité ne contient aucun élément qui ne soit pas déjà connu en matière d'évaluation de l'invalidité. Toutefois, sous l'angle de la médecine d'assurance, l'auteur tient à exprimer deux attentes, une envers le corps médical et l'autre envers les praticiens du droit, toutes deux en vue d'améliorer leur collaboration:

Dans le cadre de l'appréciation de l'incapacité de travail, les médecins sont appelés à s'exprimer plus largement sur les troubles fonctionnels, les baisses de performance et les ressources, en plus des explications relatives aux examens précédant le diagnostic médical.

Inversement, on est en droit d'attendre des praticiens du droit qu'ils justifient avec diligence, sous l'angle juridique, les motifs pour lesquels ils s'écartent de l'évaluation médicale de l'incapacité de travail ou parviennent à des conclusions différentes.

Dans cette perspective, la répartition des tâches entre médecine et droit dans le cadre de l'évaluation de l'incapacité de travail devrait s'avérer fructueuse.

\section{Hanspeter Kuhn}

Avocat, Chef de la division Service juridique FMH

Correspondance:

Hanspeter Kuhn

FMH / Division Service juridique

Elfenstrasse 18

CH-3000 Berne 15

Tél. 031351111

Fax 0313591112

lex[at]fmh.ch

\section{Commentaire juridique}

Il convient de se ranger à l'avis du Dr Renato Marelli selon lequel l'une des tâches importantes de l'expert médical est d'établir «quelles fonctions physiques et/ ou psychiques sont limitées, dans quelle mesure et de quelle manière elles le sont, et [d']en déduire quelles activités la personne assurée ne peut plus exercer ou uniquement de manière limitée.» «Doctrine et jurisprudence définissent la notion d'incapacité de travail comme une limitation physique, mentale ou psychique qui débouche sur une incapacité totale ou par- tielle d'accomplir le travail raisonnablement exigible dans la profession ou le domaine d'activité actuels [1]. Il s'ensuit dès lors que l'appréciation et l'évaluation, par un expert désigné à cette fin, des capacités et du profil de la profession relèvent de l'établissement des faits. Il incombe en principe au médecin de déterminer la capacité de travail médicale théorique [...], car celle-ci recouvre les capacités physiques et mentales [2].» Ce faisant, l'expert doit évaluer consciencieusement tous les éléments de diagnostic à la lumière de 
l'état actuel des connaissances médicales [3] scientifiques. L'étude citée par l'arrêt du Tribunal fédéral montre précisément qu'on ne peut pas toujours se fier uniquement aux méthodes d'investigation qualifiées d'objectives par les profanes, telle l'IRM. Une fixation sur les analyses de laboratoire ou sur les procédés d'imagerie reflèterait une conception réductrice de la science médicale. Le législateur lui-même a expressément consacré une conception plus large de la médecine dans l'Ordre juridique suisse. Les diplômés de médecine comprennent «les problèmes de santé de façon globale et [savent] identifier en particulier les facteurs et les conséquences de nature physique, psychique, sociale, juridique, économique, culturelle et écologique, et en [tiennent] compte dans la résolution des problèmes de santé aux niveaux individuel et collectif.» [4]

Du point de vue juridique, la phrase de l'arrêt du Tribunal fédéral (ATF) aux termes de laquelle «il n'existe pas de corrélation entre le diagnostic médical et l'incapacité de travail, et ce tant en ce qui concerne les affections somatiques qu'en ce qui concerne les affections psychiques» prête pour le moins à confusion. Les exemples contraires, dans lesquels il existe un rapport manifeste entre diagnostic et incapacité de travail, sont évidents. D’où vient dès lors ce désarroi?

Il y a lieu tout d'abord de constater que la notion d'«incapacité de travail» n'apparaît pas dans les définitions légales relatives à l'assurance invalidité:

«Est réputée invalidité l'incapacité de gain totale ou partielle qui est présumée permanente ou de longue durée.» (Art. 8 de la loi fédérale sur la partie générale du droit des assurances sociales, LPGA).

«Est réputée incapacité de gain toute diminution de l'ensemble ou d'une partie des possibilités de gain de l'assuré sur un marché du travail équilibré dans son domaine d'activité, si cette diminution résulte d'une atteinte à sa santé physique, mentale ou psychique et qu'elle persiste après les traitements et les mesures de réadaptation exigibles.» (Art. 7 LPGA).

Ensuite, l'incapacité de travail définie à l'article 6 LPGA recouvre deux réalités différentes:

«Est réputée incapacité de travail toute perte, totale ou partielle, de l'aptitude de l'assuré à accomplir dans sa profession ou son domaine d'activité le travail qui peut raisonnablement être exigé de lui, si cette perte résulte d'une atteinte à sa santé physique, mentale ou psychique. En cas d'incapacité de travail de longue durée, l'activité qui peut être exigée de lui peut aussi relever d'une autre profession ou d'un autre domaine d'activité.»

Dès lors, la proposition de formulation suivante, certes plus longue, répondrait probablement mieux à la double fonction de la notion d'incapacité de travail dans la LPGA: «il n'existe en général pas de corrélation directe entre le diagnostic médical et l'incapacité de travail, en particulier en cas d'incapacité de travail de longue durée.»

\section{Références}

1 Scartazzini G, Hürzeler M. Bundessozialversicherungsrecht, 4 e éd. Bâle 2012, p. 59 s. (traduction FMH).

2 Stolkin P. Was ist Sachverhalt und was ist Recht?, in Jusletter du 25 août 2014, note 31 (traduction FMH).

3 Dans ce sens Stolkin, op. cit., note $81:$ «[...] Il relève exclusivement de l'expert, et non du juge, de déterminer les axiomes scientifiques empiriques grâce auxquels il compte parvenir à un résultat. Il doit toutefois exposer ses conclusions de manière compréhensible pour le juge et les juristes. [...]» (traduction FMH).

4 Art. 8 let. f de la loi sur les professions médicales.

\section{Sujets} d'actualité du forum

Venez débattre avec nous! Dans la rubrique forum, nous présentons régulièrement des sujets d'actualité politique, économique et scientifique ayant trait au système de santé. Donnez votre avis ou commentez les affirmations de vos confrères. Pour accéder au forum: www.bullmed.ch/forum/ 